\title{
Brushless DC Motor Drive the Automatic Control of Clutch
}

\author{
Tianchun Lin ${ }^{1}$, Liuyi Chang ${ }^{1}$, Wangyong $\mathrm{Chao}^{1}$, Kongfan $\mathrm{Wu}^{2}$ \\ ${ }^{1}$ Mechanical and Electrical Engineering College, Changchun University of Science and Technology, \\ Changchun, Jilin \\ ${ }^{2}$ Technology Center, Jinlin Northeast Industrial Group, Changchun, Jilin
}

Keywords: Automatic Clutch; Brushless DC Motor; Controller

\begin{abstract}
Automatic clutch is on the basis of original car cancelled the clutch pedal, increased the electronic control unit. Without stepping on the clutch pedal when realize shift, it reduces the difficulty of the operation. Automatic clutch control system (ACS) with brushless DC motor is designed; this system can improve the service life of the ACS. The paper designs the corresponding DC motor controller using ATmega48 MCU.
\end{abstract}

\section{Introduction}

Automobile clutch as an important part in the transmission system; it plays the role of transferring or interrupting the power. For vehicles which is equipped with traditional mechanical gearbox (MT) ,the driver needs to step the clutch pedal when the car started or shifted, then slowly release the pedal, completed the process of clutch engagement, this may give the driver a great deal of physical exertion and ideological burden, and brings many other unsafely factors. Therefore, automatic clutch comes into being. Designed of automatic clutch adopts brushless dc motor provides the power, adopted the brushless dc motor to the vehicle starts, which can reduce the failure rate effectively, and according to actual usage, corresponding circuit control system has been designed, achieve the desired control function. Designed of automatic control of clutch in the mechanical structure achieves the features of clutch quickly disconnect and slowly joint, and adopts hydraulic actuator that make the implement results more accurate.

\section{Designed of the Control Circuit and Drive of the Brushless motor}

Brushless DC motor works. There are similar between brushless motor and brush motor in structure, both have rotor and stator, compared with brush motor, the brushless motor has the reverse structure, the brush motor rotor is coil windings, connected with power output shaft, the stator is a permanent magnetic steel; the brushless motor rotor is permanent magnetic steel, together with the housing and are connected with output shaft, the stator is the windings, so brushless motor is that removed the commutation brush of brush motor for alternating the electromagnetic fields ${ }^{[1]}$.

In simple terms, by changing the current wave alternating frequency and waveform that input to the brushless motor stator coils, formed a magnetic field that rotates around the geometric axis of the motor surround the windings ,this magnetic field drives the permanent magnetic steel spin that is on the rotor, then the motor turns up, the performance of the motor related to the number of magnet 、magnet flux intensity 、input voltage and other factors, and more related to the brushless control function.

Designed of Brushless motor controller.Figure 1 shows the principle diagram of three-phase brushless motor controller by using ATmega 48 SCM. Among them,PC0、 PC1 and PC2 as the input, applied receive the hall sensor detection signals that comes from the commutation of the motor $\mathrm{U}, \mathrm{V}, \mathrm{W}$; PD5 and PD6 are applied to control the power drive device of the motor phase U; PB1 and PB2 are applied to control the power drive device of the motor phase V; PD3 and PB3 are applied to control power drive device of the motor phase $\mathrm{W}$; the rotor speed of brushless dc motor is influenced by the speed of the rotating magnetic speed of the stator and the number of rotor poles $^{[2]}$. When the number of rotor poles is fixed, just changed the frequency of the rotating magnetic field of the stator, then the rotor speed has been changed. Brushless dc motor is that 
controls the rotating magnetic field of the stator, and make the rotor speed of the motor feedback to the control center so that it can be corrected repeatedly, in order to close to the characteristics of the dc motor. When the load has been changed, it can control the motor rotor within the range of the rated load maintain a certain speed.

Figure 2 shows the window comparator circuit diagram composed by two voltage comparators. When the compared signal voltage $U_{\text {in }}$ is located between the threshold voltage $\left(U_{R 1}<U_{\text {in }}<U_{R 2}\right)$, the output is high level. When it is not located between the threshold voltage $\left(U_{\text {in }}<U_{R 1}\right.$ or $\left.U_{\text {in }}>U_{R 2}\right)$, the output is low level, the window voltage is $\Delta \mathrm{U}=\mathrm{U}_{\mathrm{R} 2}-\mathrm{U}_{\mathrm{R} 1}$.It can be used to determine whether the input signal level is located between the specified threshold level.

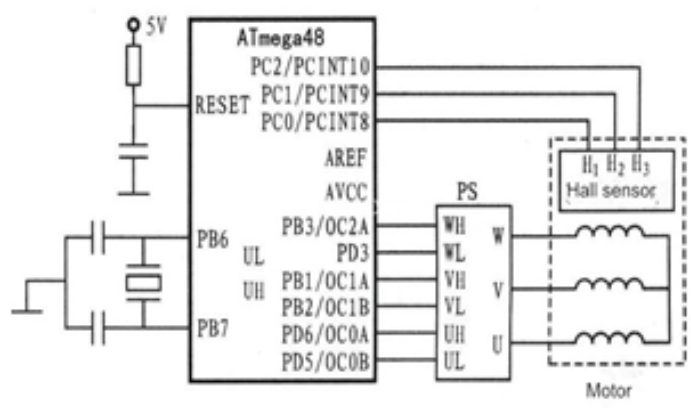

Figure 1 three-phase brushless motor controller is realized by using ATmega48 schematic diagram

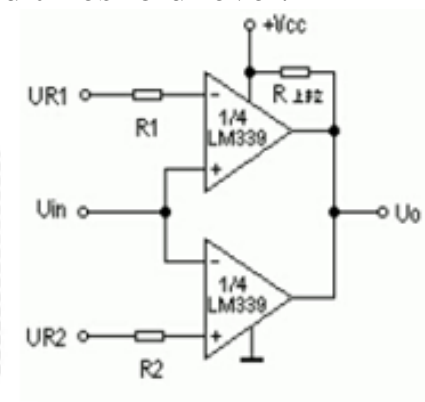

(a)

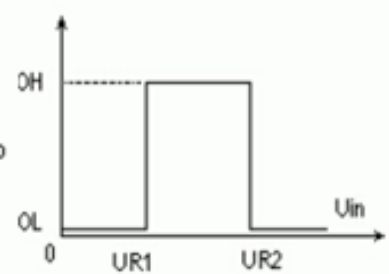

(b)

Figure 2 window comparator circuit diagrams

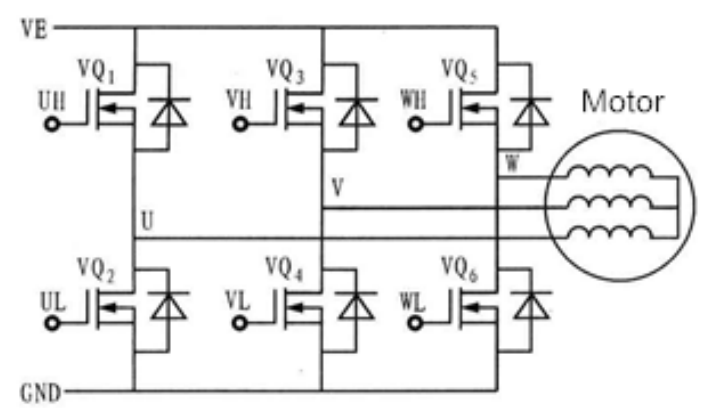

Figure 3 power drive circuit

Designed of brushless motor drive.Figure 3 shows the power drive circuit principle diagram. The drive part is composed by six power transistors of the upper arm VQ1, VQ3, VQ5and the lower arm VQ2, VQ4, VQ6, which is used for connecting the motor as a switch of controlling through the motor windings. The control section provides PWM, applied to decision the power transistor switch frequency and the moment of commutation. When the speed of brushless dc motor is controlled, generally hope that when the load changed also make the motor speed stability within the set value, and without too much volatility ${ }^{[3]}$.Therefore, the hall sensor disposed inside of the brushless motor to sense the magnetic field changes, the sensor not only can be used as the speed feedback parts of the motor speed in closed-loop control but also can be used as the basis of the phase sequence control.

When the controller is working, according to the hall sensor can detect the current position of the motor rotor, according to the stator wingdings decide to open or close the sequence of the power transistor, make the current flows the motor windings sequentially, to produce a rotation magnetic field of forward or reverse, and interact with the rotor magnet, make the motor rotates clockwise or counterclockwise. When the motor rotor rotated to the position of next signals that the hall sensor can detect, and then open the next set of the power transistors, so repeatedly, the motor can rotate based on the same direction, until the controller decides to make the motor rotor stopped, and then turn off the power transistor; when the motor rotor is on the reverse direction, then turn on the power transistor, but in the reverse order. PWM is to determine the way of the motor speed fast or slow, how to generate PWM is the core of realizing accurate control speed. 


\section{Mechanical Design}

Designed of Mechanism.Figure 4 shows the automatic control of clutch principle diagram of mechanical structure design ,among them, rocker 9, putter 1, piston 2 and hydraulic cylinder 3 composed the hydraulic actuator of the clutch, they are the executor of the clutch action, controlling the clutch automatic separation and smooth engagement. The function of the actuator is tracking the clutch engagement law; the actuator control performance whether is good or bad has an important influence on the comfort of the vehicles, the economy of the fuel and the lifetime of vehicles $^{[4]}$.
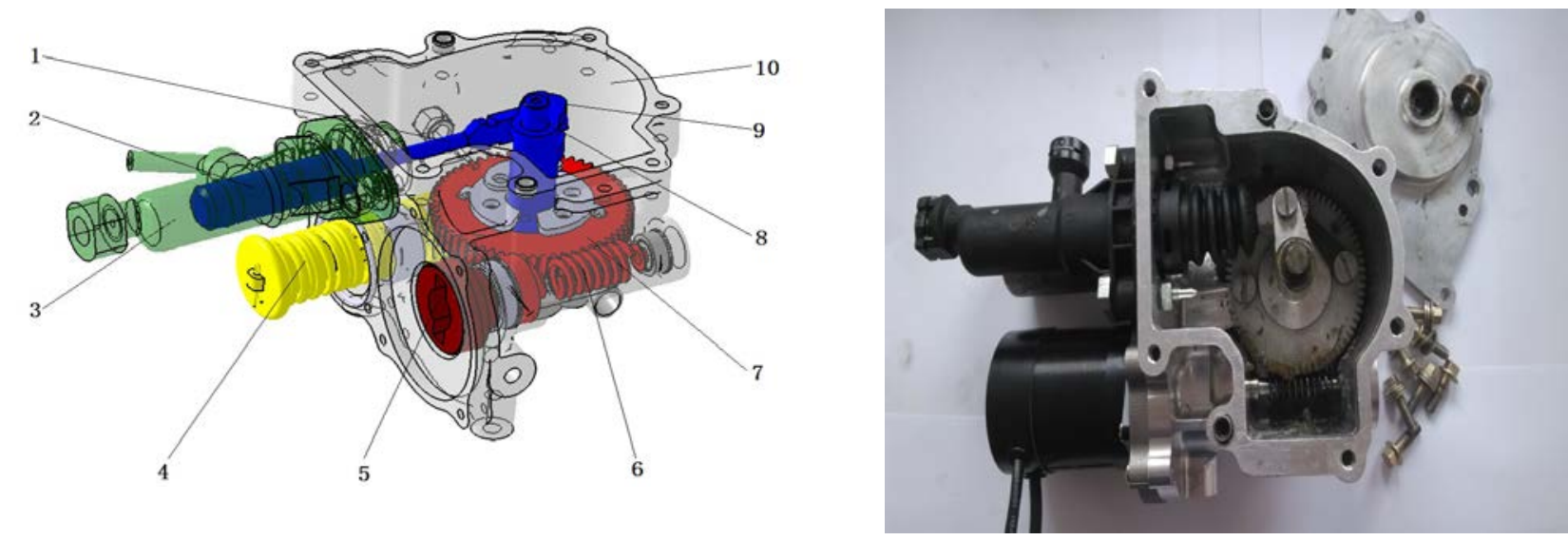

1.putter 2.piston 3.hydraulic cylinder 4.aid agencies 5.couplings

6.worm 7.worm gear 8.worm gear shaft 9.rocker 10.housing

Figure 4 automatic control clutch principle diagram and physical map of the mechanical structure

From the diagram, the part of 4 is the aid agency of the actuator, which is made up of spring and putter. When the worm gear shaft 9 turns to drive the actuator movement, in aid agency, the spring is compressed, then achieved the process that actuator controls the clutch quickly disconnect and slowly join.

From the diagram, couplings 5 realized the dc motor input of the power, worm 6 and worm gear 7 achieved power transmission, the housing 10 plays role in supporting and sealing.

The major feature of the mechanical design section is the putter、spring aid agency on the worm can achieve the process of clutch quickly disconnect and slowly join. Hydraulic switch actuator has the advantage of large capacity, high control accuracy, fast response and easy to spatial arrangement ${ }^{[5]}$.

Stress Analysis.As shown in figure 5, simplified and reconstructed the worm and worm gear and its transmission lever, and had a stress analysis. When analysis, each component was in the maximum force movement point. The size of each load was: the worm torque is $400 \mathrm{Nmm}$, transmission lever fulcrum force is $1000 \mathrm{~N}$. Except the worm material is nylon, other parts are made up of mile steel material.

The component had a static stress simulation on the INVENTOR environment platform, the results are shown in Figure 5.

The maximum stress appeared in the lower part of the rod as shown in Figure 5 position, the result was $53.3 \mathrm{Mpa}$, below the material(mild steel)yield strength. Each part safety coefficient is between 3.88 and 15. From the analysis results, this design has confirmed the requirement of the required strength.

\section{Conclusions}

This designs of automatic control clutch that adopts brushless dc motor drives automatic clutch system(ACS), which can be able to improve the service life of the system, reduce the failure rate. Designed of brushless dc motor electric control system applied in vehicles with an automatic clutch, make the control process more accurate. Designed of clutch mechanical structure, the putter s spring 
auxiliary actuator on the worm can achieve quickly disconnect and slowly join of the clutch. The hydraulic switch actuator has the advantage of large capacity , high control accuracy 、 fast response speed and ease of spatial arrangement.
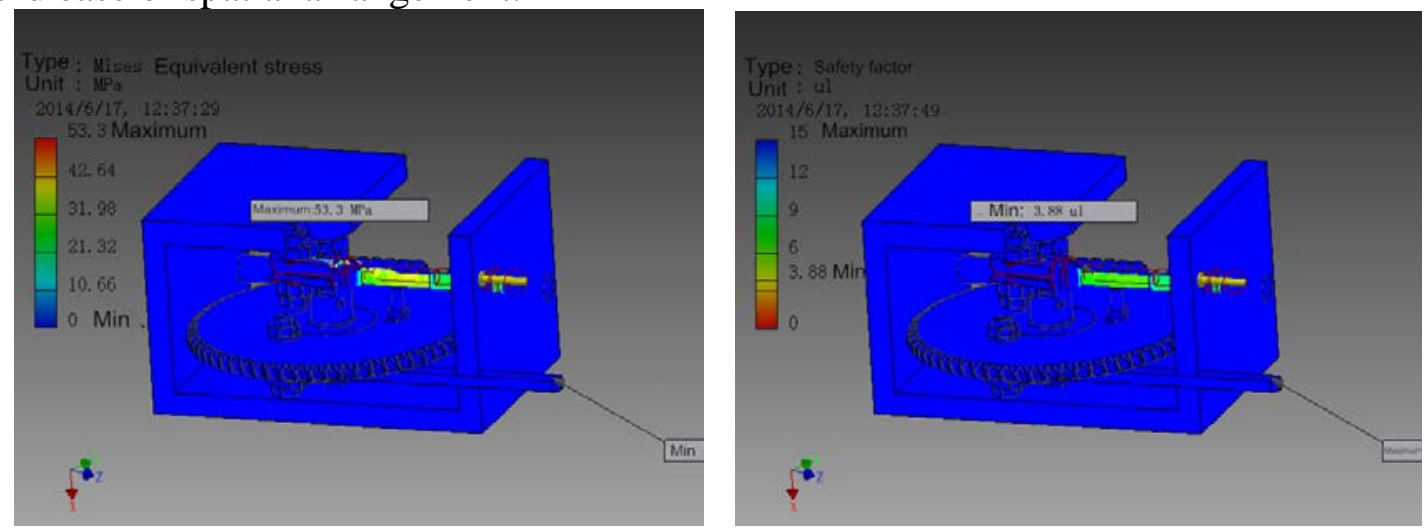

Figure 5 Stress analysis diagram

\section{References}

[1] Dingzhi Gang. Research and Development Progress of Brushless Dc Motor. Micro-motor, 2003,33 (1):29, 21-24, 43.

[2] QiRong. Research Brushless DC Motor PWM Modulation Method and the Relationship between the Torque Ripple.Micro-motor, 2006.39.

[3] Chenguo Cheng. PWM Frequency Control Technology. Machinery Industry Press,1999.

[4] Leiyu Long .The Research of Improving the Electronically Controlled Automatic Transmission Mechanical Properties[D].Jilin University of Technology, ChangChun,1998.

[5] XuFan. Design and Development of Automated Clutch Actuator[D]. Shanghai Jiaotong University, ShangHai,2006. 